\title{
Overwhelming Postsplenectomy Infection for Splenectomized HCV Patients
}

\author{
Naoki Hashimoto \\ School of Medicine, Kinki University, Osaka, Japan \\ Email: gojigen000@gmail.com
}

Received 3 July 2015; accepted 19 July 2015; published 24 July 2015

Copyright (C) 2015 by author and OALib.

This work is licensed under the Creative Commons Attribution International License (CC BY). http://creativecommons.org/licenses/by/4.0/

(c) (i) Open Access

\begin{abstract}
Pegylated-interferon (IFN) plus ribavirin remains the most effective therapeutic regimen for patients with chronic hepatitis $C$ interferon. Thrombocytopenia is a common side effect of this treatment, often leading to discontinuation of a potentially curative therapy. Splenectomy was a clinically effective treatment for hepatitis $C$ virus-associated thrombocytopenia. Splenectomy in patients with hepatitis $C$ cirrhosis is now safer prelude to antiviral treatment. Overwhelming post splenectomy infection (OPSI) syndrome is a rare condition, but is associated with high mortality. However, recognition and clinical management of OPSI is not well established. We reviewed the literature characterizing the clinicopathological features of OPSI and assessed the most effective and feasible administration of the condition. Prevention strategies such as vaccination and education are also potentially important parts of the strategy for splenectomized HCV patients.
\end{abstract}

\section{Keywords}

Chronic Hepatitis C, Interferon, Splenectomy, OPSI

Subject Areas: Emergency \& Critical Care, Surgery \& Surgical Specialties

\section{Introduction}

The world Health organization has estimated that 170 million people, or $3 \%$ of the world's population, are infected with hepatitis C virus (HCV) [1] with the highest prevalence rates noted in Africa and Asia. In the United States, the incidence of HCV infection is declining secondary to effective blood donor screening adopted in the early 1990s and changing practices of intravenous drug users due to an increased awareness of HIV (Human Immunodeficiency Virus) and hepatitis. Several factors influence the rate of progression of hepatitis C. Progression is hastened when acquired via blood transfusion; at an older age; in males; in non-African-Americans; in the setting of excessive alcohol ( and possibly tobacco) use; and in persons with HIV and /or HBV (Hepatitis B Virus) coinfection or feature of metabolic syndrome. Although acute HCV infection resolves spontaneously in 
some cases, the virus establishes chronic infection in 55\% - 85\% of infected individuals and persists for decades. Left untreated, approximately $20 \%$ of chronically infected individuals will go on to develop cirrhosis and $1 \%-5 \%$ will develop liver failure and hepatocellular carcinoma. HCV infection remains a common cause of chronic liver disease and is an increasing indication for liver transplantation. Initial treatment has typically consisted of a combination of interferon (INF) $\alpha$-2b and ribavirin for 48 weeks [2]. The addition of a polyethylene glycol (pegylation) moiety to IFN improves the pharmacokinetics of this protein-based therapy allowing for a longer halflife and once-a-week dosing intervals [3]. Recent multicenter trials have demonstrated the superiority of pegylated IFN plus rivavirin compared to pegylated IFN alone or non-pegylated combination therapy [4]. Yet, patients with severe baseline thrombocytopenia may not be candidates for therapy due to the risk of exacerbation by IFN. Strict adherence to combination therapy for HCV enhances the likelihood of inducing an initial virological response, and adherence beyond 12 - 24 weeks strongly benefits those with an initial response [5]. For patients with low baseline platelet counts, a full course of therapy is frequency not possible due to the development of severe IFN induced thrombocytopenia.

\section{Interferon}

Although sustained viral response after treatment with interferon alfa monotherapy was $10 \%-15 \%$, the addition of ribavirin significantly improved the response rated to 30\% - 40\% [6]. Most recently, the use of pegylated interferon has further improved response rate, and more than half of the treated patients can expert to have a sustained response with combination therapy using peginterferon and ribavirin [7]. However, leukopenia and / or thrombocytopenia are the major determinant factor for completing interferon treatment, because such hepatitis $\mathrm{C}$ infected patient are often suffering from hypersplenism [8]. In addition to a low baseline platelet count in some patients with chronic HCV infection, the use of IFN to treat HCV can exacerbate the thrombocytopenia. Several mechanisms for this side effect have been described. The most common reason for a drop in platelet count is IFN-induced pancytopenia resulting from bone marrow suppression and the direct inhibitory effect of IFN on megakaryocytes. Platelet counts may decrease by as much as $25 \%$ to $50 \%$ from baseline [9]. This effect is dose dependent, usually occurs within the first few weeks of IFN therapy, and leads to dose reductions or withdrawal from therapy in up to $25 \%$ of patients [10]. Because interferon treatment is the only critical treatment that eliminate hepatitis $C$ virus, reduces fibrosis stage, and decrease the risk of hepatocellular carcinoma, medical or surgical reversal of hypersplenism is necessary.

\section{PSE, Splenectomy}

Partial splenic embolization (PSE) is a non-surgical procedure developed to treat hypersplenism as a result of hepatic disease. In 1973, Maddison [11] proposed splenic artery embolization for the treatment of hypersplenism. Initially, total ablation of the splenic parenchyma was performed, but severe complications, including splenic abscess, serious pneumonia, sepsis, hematoma and death. Then, Spigos et al. [12] developed a technique for PSE, allowing vascular occlusion to be performed safely and effectively.

Recent advances in interventional radiology (IVR) have decreased the side-effects of PSE and greatly expanded the indications.

Although there have been similar reports of splenic embolization for interferon therapy, prolonged high fever and pain due to massive splenic infarction are the most common unpleasant side effects after splenic embolization [13]. In contrast, surgical splenectomy renders patients free from such annoying complications. Moreover, the improvements in pancytopenia after splenectomy last for significantly longer periods, almost indefinitely, compared with PSE. Hayashi et al. reported that effective splenectomy for patients receiving interferon treatments without complications; increase the platelet count from $3.2 \times 10 / \mathrm{mm}^{3}$ to $22.2 \times 10 / \mathrm{mm}^{3}$ with median hospital stay of 9 days after surgery. Moreover, with the recent advancements in laparoscopic techniques, Kercher [14] et al. reported that laparoscopic splenectomy was performed for 11 patients with mean blood loss of $141 \mathrm{ml}$ and mean operative time of $189 \mathrm{~min}$. They reported that platelet count increased from $5.5 \times 10^{4} / \mathrm{mm}^{3}$ to $43.9 \times$ $10^{4} / \mathrm{mm}^{3}$ after splenectomy, and was maintained at over $10 \times 104 / \mathrm{mm}^{3}$ during subsequent pegylated interferon therapy.

The mean postoperative platelet count after splenectomy was more than six times the preoperative mean and has been maintained for up to 18 months after splenectomy. 


\section{OPSI}

Although there are favorable outcomes of splenectomy in chronic hepatic disorders, there are still a few concerns that need to be overcome; overwhelming post-splenectomysepsis (OPSS). The first description of OPSI was published by King and Schumaker in 1952 [15]. The disease may begin as a minor flu like illness that rapidly escalates into a fulminant infection. Initial symptoms are often mild, with an influenza-like presentation that includes fever, malaise, headache, vomiting, diarrhea and abdominal pain. It is most common in the first two years after splenectomy but may occur decades later [16]. OPSS can be serious fulminant septic process with a high mortality rate (50\% - 70\%) despite aggressive therapy (intravenous fluids, antibiotics, vasopressors, steroids, heparin, packed red blood cells, platelets, cryoprecipitates, and freshfrozen plasma) after splenectomy. Pneumonia and meningitis are frequent more severe concomitants. The clinical course may rapidly progress to coma and death within 24 to $48 \mathrm{~h}$, due to the high incidence of shock, hypoglycemia, marked acidosis, electrolyte abnormalities, respiratory distress, and disseminated intravascular coagulation (DIC).

It has been well documented, in the setting of splenectomy in pediatric populations, in hematologic/autoimmune disorders, or those with asplenia. Infants (under the age of 2 years) do not acquire specific antibodies against encapsulated organisms until relatively late. Patients with hematological and autoimmune disorders or lymphoma show impaired immunity. Both groups may have an increased susceptibility for OPSI. While liver kupffer cells clear most well-opsonized bacteria, encapsulated organisms are removed by the spleen. Therefore, patients who are asplenic are at lifelong risk for a variety of serious infections, especially encapsulated organisms such as Streptococcus pneumonia, Neisseria meningitis and Hemophilus influenza. Streptococcus pneumonia constitutes $70 \%$ - 90\% of these OPSIs. Neisseria meningitis and hemophilus influenza constitute most of the rest.

Therefore, not only vaccinations for those pathogens but also extensive patient education for their easy infectivity is highly recommended before splenectomy [17].

\section{Prevention of OPSI}

Jockovich [18] reported no OPSI among patients vaccinated before splenectomy, however, $10.4 \%$ of patients who did not receive vaccination developed OPSI. In addition, $5 \%$ of patients who were given vaccination after splenectomy developed OPSI. For elective splenectomy, the vaccine should be given at least 2 week before surgery [19]. Vaccines available for the most common organisms include the 23-valent pneumococcal polysaccharide vaccine, the Hemophilus influenza type B vaccine and the Meningococcal vaccine [20]. Pneumococcal vaccine (Pneumovax, Banyu pharmaceutical, Japan) is approved before splenectomy by the Japanese insurance system. Established the new evidence, for the need of vaccination after splenectomy for chronic liver disorders, is anticipated.

Finally, patient education represents a mandatory strategy for preventing OPSI. Studies have shown that from $11 \%$ to $50 \%$ of splenecomized patients remain unaware of their increased risk for serious infection or the appropriate health precaution that should be undertaken [21]. This is our patient interview data (Table 1). Most of patients did not receive a specific vaccine after splenectomy, did not know that an asplenic stat leads to a greater risk for life-threatening infection and would not seek medical advice urgently in case of fever. No patients knew that special precaution should be taken when traveling.

Patients need to know the nature and likelihood of overwhelming post-splenectomy infection and they need to know that they should seek medical attention if they become ill and feverish. Each patient should carry at all times a letter or card documenting the splenectomy and should wear a bracelet or pendant to signal their status to doctors and other healthcare staff. Healthcare records in hospital and primary care should clearly record that these patients have had splenectomy. Primary care doctors, who may be responsible for keeping patients fully immunized, should know what vaccinations these patients have had. The role and efficacy of antibacterial prophylaxis remains unclear, and not all countries recommend it. Some studies show that patients may not adhere to prophylaxis but, in the absence of robust systematic evidence against this approach, the chief medical officer in the United Kingdom recommends lifelong prophylaxis with oral phenoxymethyl penicillin (or erythromycin for people who are allergic to penicillin). Patients who develop infection despite vaccination and antibacterial prophylaxis should go into hospital for treatment with broad spectrum systemic antibiotics. In addition, patients might keep a supply of antibiotics at home, changing from prophylactic to therapeutic doses if they develop febrile illness. This is particularly important for those who do not, or will not, take prophylaxis, a group 
Table 1. Questions administered to splenectomized patients.

1. Are you aware you had your spleen removed?

Yes: $100 \%$ no: $0 \%$

2. Do you think it increases your chances of certain infection?

Yes: $25 \%$ no: $75 \%$

3. Did you receive any vaccinations within a month before surgery?

Yes: $0 \%$ no: $100 \%$

4. Are you taking oral penicillin?

Yes: $0 \%$ no: $100 \%$

5. How long will you take the oral penicillin?

Yes: $0 \%$ no: $100 \%$

6. What would you do if you got sick or developed fever?

Would you take a full dose of antibiotics orb visit your health-care physician?

Yes: $88 \%$ no: $12 \%$

7. Do you know the name of your antibiotics?

Yes: $0 \%$ no: $100 \%$

8. Do you have up-to-date full dose antibiotics at home?

Yes: $25 \%$ no: $75 \%$

9. What would you do if you got a scratch or small dog-bite? Would you visit your health care

Physician?

Yes: $37 \%$ no: $73 \%$

10. How did you get your information about all this?

Yes: $75 \%$ no: $25 \%$

whom doctors should make more aware of the risk and dangers of infection.

\section{Conclusion}

Education of HCV with recently introduced therapy of pegylated interferon and rivavirin would be particularly beneficial in those with advanced liver fibrosis or cirrhosis. Splenectomy might also be useful to raise platelet counts so that pegylated interferon based therapy can be performed safely in patients with cirrhosis due to HCV. Splenectomy results in an increased risk of septic complications associated with a high mortality rate, the most serious being the development of OPSI. Prevention strategies such as vaccination and education are also potentially important parts of the strategy for splenectomized HCV patients.

\section{References}

[1] Anonymous (1999) Hepatitis C-Global Prevalence (Update). World Health Organization Weekly Epidemiological Record, 74, 425-427.

[2] McHutchison, J.G., Goodman, Z.D., Ling, M.H., et al. (1998) Interferon Alfa-2b Alone or in Combination with Ribavirin as Initial Treatment for Chronic Hepatitis C. Hepatitis Interventional Therapy Group. The New England Journal of Medicine, 339, 1485-1492. http://dx.doi.org/10.1056/NEJM199811193392101

[3] Youngster, S., Wang, Y.S., Grace, M., et al. (2002) Structure, Biology and Therapeutic Implications of Pegylated Interferon Alpha-2b. Current Pharmaceutical Design, 8, 2139-2157. http://dx.doi.org/10.2174/1381612023393242 
[4] Fried, M.W., Shiffman, M.L., Reddy, K.R., et al. (2002) Peginterferon Alfa-2a plus Ribavirin for Chronic Hepatitis C Virus Infection. The New England Journal of Medicine, 347, 975-982. http://dx.doi.org/10.1056/NEJMoa020047

[5] McHutchison, J.G., Manns, M., Patel, K., et al. (2002) Adherence to Combination Therapy Enhances Sustained Response in Genotype-1-Infected Patients with Chronic Hepatitis C. Gastroenterology, 123, 1061-1069. http://dx.doi.org/10.1053/gast.2002.35950

[6] Kamal, S.M., EI Kamary, S.S., Shardel, M.D., et al. (2007) Pegylated Interferon Alpha-2b plus Ribavirin in Patients with Genotype 4 Chronic Hepatitis C: The Role of Rapid and Early Virologic Response. Hepatology, 46, 1732-1740. http://dx.doi.org/10.1002/hep.21917

[7] Di Bisceglie, A.M. and Hoofnagle, J.H. (2002) Optimal Therapy of Hepatitis C. Hepatology, 36, S121-127. http://dx.doi.org/10.1002/hep.1840360716

[8] Hayashi, P.H., Mehia, C., Joachim Reimers, H., et al. (2006) Splenectomy for Thrombocytopenia in Patients with Hepatitis C Cirrhosis. Journal of Clinical Gastroenterology, 40, 740-744. http://dx.doi.org/10.1097/00004836-200609000-00015

[9] Renault, P.F. and Hoofnagle, J.H. (1989) Side Effects of Alpha Interferon. Seminars in Liver Disease, 9, $273-277$. http://dx.doi.org/10.1055/s-2008-1040523

[10] Martin, T.G. and Shuman, M.A. (1998) Interferon-Induced Thrombocytopenia; It Is Time for Thrombopoietin. Hepatology, 28, 1430-1432. http://dx.doi.org/10.1002/hep.510280536

[11] Maddison, F.E. (1973) Embolic Therapy of Hypersplenism. Investigative Radiology, 8, $280-281$. http://dx.doi.org/10.1097/00004424-197307000-00054

[12] Moges, M.F., Spigos, D.G., Pollak, R., Abejo, R., Pavel, D.G., Tan, W.S. and Jonasson, O. (1984) Partial Splenic Embolization, an Alternative to Splenectomy-Results of a Prospective, Randomized Study. Surgery, 96, 694-702.

[13] Pålsson, B. and Verbaan, H. (2005) Partial Splenic Embolization as Pretreatment for Antiviral Therapy in Hepatitis C Virus Infection. European Journal of Gastroenterology \& Hepatology, 17, 1157-1164.

[14] Kercher, K.W., Carbonell, A.M., Heniford, B.T., Matthews, B.D., Cunningham, D.M. and Reindollar, R.W. (2004) Laparoscopic Splenectomy Reverses Thrombocytopenia in Patients with Hepatitis C Cirrhosis and Portal Hypertension. Journal of Gastrointestinal Surgery, 8, 120-126. http://dx.doi.org/10.1016/j.gassur.2003.10.009

[15] King, H., Shumacker Jr., H.B. (1952) Splenic Studies: I. Susceptibility to Infection after Splenectomy Performed Infancy. Annals of Surgery, 136, 239-242. http://dx.doi.org/10.1097/00000658-195208000-00006

[16] Evans, D.I. (1985) Postsplenectomy Sepsis 10 Years or More after Operation. Journal of Clinical Pathology, 38, 309311. http://dx.doi.org/10.1136/jcp.38.3.309

[17] Cullingford, G.L., Watkins, D.N., Watts, A.D. and Mallon, D.F. (1991) Severe Late Postsplenectomy Infection. British Journal of Surgery, 78, 716-721. http://dx.doi.org/10.1002/bjs.1800780626

[18] Jockovich, M., Mendenhall, N.P., Somebeck, M.D., Talbert, J.L., Copeland III, E.M. and Bland, K.I. (1994) LongTerm Complications of Laparotomy in Hodgkin's Disease. Annals of Surgery, 219, 615-621. http://dx.doi.org/10.1097/00000658-199406000-00004

[19] Landgren, O., Bjorkholm, M., konradsen, H.B., Söderqvist, M., Nilsson, B. and Gustavsson, A. (2004) A Prospective Study on Antibody Response to Repeated Vaccinations with Pneumococcal Capsular Polysaccharide in Splentomized Individuals with Special Reference to Hodgkin’s Lymphoma. Journal of Internal Medicine, 255, 664-673. http://dx.doi.org/10.1111/j.1365-2796.2004.01312.x

[20] Shatz, D.V. (2005) Vaccination Considerations in the Asplenic Patient. Expert Review of Vaccines, 4, 27-34. http://dx.doi.org/10.1586/14760584.4.1.27

[21] White, K.S., Covington, D., Churchill, P., Maxwell, J.G., Norman, K.S. and Clancy, T.V. (1991) Patient Awareness of Health Precautions after Splenectomy. American Journal of Infection Control, 19, 36-41. http://dx.doi.org/10.1016/0196-6553(91)90158-9 